\title{
La transversalización del enfoque de géneros en las políticas culturales públicas: el caso del Ministerio de Cultura argentino ${ }^{1}$
}

\section{The mainstreaming of the gender approach in public cultural policies: the case of the Ministry of Culture argentine}

\section{Marcela País Andrade}

\author{
Marcela País Andrade es investigadora adjunta del Consejo Nacional de \\ Investigaciones Científicas y Técnicas y profesora en la Universidad de \\ Buenos Aires, Argentina. \\ E-mail: maky2007@gmail.com
}

\section{resumen}

Desde un enfoque socioantropológico se problematizan las relaciones entre las políticas culturales y el Estado en las formas de diseñar e implementar la transversalización de los enfoques de géneros/feminismos. La propuesta se ejemplifica observando la gestión de algunas líneas de acción circunscritas en el Ministerio de Cultura de la Nación Argentina entre los años 2014 y 2015. Para ello: se explica la vinculación del campo cultural con el contexto político/económico/social a nivel regional/local que contextualizan las nociones de desarrollo y diversidad cultural; se describen las posibilidades/limitaciones de los enfoques de géneros/feminismos que se observa en la política cultural pública reciente a través de sus planes y/o programas que enmarcaron la agencia de los sujetos inmersos en ellos. Por último, se señala la falta de integración en la gestión interna para visibilizar la necesidad de una planificación estratégica en la transversalización de los enfoques de géneros/feminismos en el campo cultural.

\section{summary}

From a socio-anthropological approach, are problematized the relationships between cultural policies and the State in the ways of designing and implementing the mainstreaming of gender / feminism approaches. The proposal is exemplified by observing the management of some lines of action circumscribed in the Ministry of Culture of the Argentine Nation between 2014 and 2015. For this purpose: is explained the link between the cultural field and the political / economic / social context at the regional / local level, that contextualize the notions of development and cultural diversity; describes the possibilities / limitations of the gender / feminism approaches observed in recent public cultural policy through their plans and / or programs that framed the agency of the subjects immersed in them; is indicated the lack of integration in internal management for to make visible the need for strategic planning in the mainstreaming of genre / feminism approaches in the cultural field.

\section{palabras clave}

política pública / cultura / género / diversidad / desarrollo

\section{keywords}

public policie / culture / gender / diversity / development 


\section{Introducción}

El estudio de la "cuestión del género" y su imbricación con las políticas públicas viene siendo observado hace ya décadas en la Argentina. Es sabido que luego de la última dictadura militar argentina (24 de marzo de 1976-10 de diciembre de 1983) nuestro país inició lentamente -no sin complejidades- el período de transición democrática. ${ }^{3}$ En este contexto, aparecen diversas demandas individuales y colectivas de mujeres referidas a problemáticas específicas de participación y de derechos ciudadanos que reclamaban ingresar en las agendas públicas. Así, a partir de los años 80 , las mujeres como colectivo retoman y profundizan temas/problemas que se habían comenzado a visibilizar en décadas anteriores y que la dictadura militar había logrado silenciar: la participación de las mujeres en los ámbitos universitarios y políticos, el ingreso al mercado de trabajo, etc. Estas transformaciones daban cuenta de mujeres que no sólo querían ocupar los espacios públicos sino producir prácticas y generar decisiones propias alejadas de los roles tradicionales asociados a la familia y a la maternidad. Comienzan a resignificarse entonces temas/problemas públicos vinculados a las mujeres/géneros ${ }^{4} /$ feminismos $^{5}$ en los ámbitos sociales, de la salud, de la reproducción, del trabajo, de la educación y por supuesto también del campo cultural.

En este sentido, existe una vasta bibliografía que aborda el diseño, la implementación y los aciertos/desaciertos de políticas públicas transversalizadas por estos enfoques. Dichos trabajos nos permiten observar cómo -en el proceso de transición democrática bajo la presidencia de Raúl Alfonsín (1983-1989)- los ámbitos sociales, culturales y políticos se caracterizaron por un despertar esperanzador en relación con la conquista de los derechos de las mujeres; por ejemplo, en 1985 se comienzan a realizar los Encuentros Nacionales de Mujeres (ENM) dando cuenta de un contexto que posibilitaba la participación de las mujeres en el campo social, cultural y político argentino (Aguilar, 2011; Jelin, 1990;Anzorena, 2013, entre otros/as).

Muy a pesar de lo logrado en la década anterior, en el transcurrir de los años 90, con el Dr. Carlos Menem en la presidencia, se van a reconfigurar los vínculos entre las mujeres y el Estado ubicándolas en la atención de los/as menos favorecidos/ as en un contexto de políticas neoliberales que caracterizaron las desigualdades y las condiciones de vida empobrecidas de dicha década generando diferentes políticas públicas focalizadas hacia las mujeres (Di Marco, 1997; Grassi, 2003, 2009; Falquet, 2003; Rodríguez Gustá y Caminotti, 2010, entre otros/as). Se llega así al inicio del nuevo siglo, marcado por una crisis económica, social y política (el mandato presidencial a cargo del Dr. Fernando de la Rúa comienza el 10 de diciembre de 1999 finalizando abruptamente el 21 de diciembre de 2001) que profundizará las desigualdades de todo el pueblo argentino. Las mujeres serán de los grupos sociales más castigados, pero también se las visibilizará en el espacio público por medio de fuertes demandas sociales/culturales y sobre todo de participación política $^{6}$ (Di Marco, 2003; Rauber, 2002; Merchán, 2001, entre otros/as). Es en esta década cuando se comienza a configurar el proceso político de origen peronista denominado kirchnerismo. ${ }^{7}$ 
En ese marco, se delinearon ciertos programas, proyectos y líneas de acción caracterizados en términos generales, por discursos que rechazaban las miradas del neoliberalismo, las políticas económicas desarrollistas y los tratados del libre comercio. Se promulgaron (con matices, por supuesto) políticas públicas destinadas a la defensa del Mercosur, el alineamiento internacional latinoamericano y la revalorización de los derechos humanos. En esta última línea, se reivindicaron ciertas acciones y/o programas gubernamentales garantes de derechos a las minorías vulneradas como grupos migrantes, indígenas, juventudes, mujeres, diversidades sexuales, $\mathrm{LGBTTTI}^{8}$, entre otros. Ahora bien, el 10 de diciembre del año 2015 asumió la presidencia de la Nación Argentina el Ingeniero Mauricio Macri representando a la coalición política Cambiemos. La vigente gestión comienza a implementar acciones políticas, económicas y socioculturales enmarcadas en discursos con tintes liberales, desarrollistas y conservadores. Actualmente, el cambio del proyecto de Nación parecería estar reconfigurando la perspectiva de géneros que transversalizaba (con matices y dudas, por cierto) tangibles líneas de acción que se venían gestando y/o desplegando durante el período anterior.

Dicho en forma breve, las complejidades en la gestión de las políticas sociales y culturales dirigidas a las mujeres a través de los diversos proyectos, planes, y/o programas se fueron configurando -a partir de la vuelta a la democracia en nuestra nación- entre las paradojas que vinculan a la sociedad civil y el Estado; a la economía y la política; al cuerpo y la política; a la distribución y el reconocimiento; a las dimensiones estructurales y las transformaciones históricas; a lo instituido y a lo instituyente en escenarios inestables y ambiguos ${ }^{9}$ (Anzorena, 2013). Estas complejidades han invisibilizado de formas particulares los vínculos entre las políticas culturales públicas y los enfoques de mujeres/géneros/feminismos. ${ }^{10}$

Por lo cual, atento a los objetivos propuestos en el proyecto más amplio del cual se desprende este artículo, aquí nos preguntamos: ¿cómo se ha ido (re)configurando contextualmente la transversalización de los enfoques de mujeres/géneros/ feminismos en las políticas culturales públicas ${ }^{11}$-mundiales/regionales/localesque surgieron para dar respuesta a las demandas socioculturales de los diversos colectivos en las últimas décadas? y ¿cómo se ha gestionado la transversalización de los enfoques de mujeres/géneros/feminismos, en y desde las políticas culturales públicas locales, para la (re)configuración de los derechos de y desde la diversidad cultural /agencias ${ }^{12}$ en cuestiones de géneros?

Para explicar lo dicho, han sido de gran aporte los textos abocados a la temática que se enfocan en el análisis del impacto de las políticas públicas, naturalizando el binomio poblaciones/políticas o - por el contrario- tensionándolo, en tanto términos necesariamente implicados y en conflicto. No obstante, la complejidad planteada requirió que problematicemos y/o deconstruyamos de manera crítica los procesos de diversidad cultural/agencias que se van conformando en relación a los géneros en el marco de las gestiones de los distintos programas, proyectos y líneas de acción gubernamentales. Así, la antropología política y feminista nos aportó la posibilidad de interpretar el mundo de estudio a través del enfoque etnográfico (Abéles, 1997; Wedel et al., 2005) el cual nos habilitó la deconstrucción de las 
aparentes totalidades racionales que son las políticas, a través de la descripción y el análisis de la compleja trama de relaciones de poder, resistencia y negociación que se tejen a nivel de la vida cotidiana (Shore y Wright, 1997). Siguiendo a Cris Shore podemos decir que un estudio antropológico de las políticas públicas se pregunta: "¿qué quiere decir política pública en este contexto? ¿Qué funciones tiene? ¿Qué intereses promueve? ¿Cuáles son sus efectos sociales? ¿Y cómo este concepto de política pública se relaciona con otros conceptos, normas o instituciones dentro de una sociedad en particular?" (2010: 29). De aquí que cuestionamos a las políticas públicas desde las poblaciones-problema que las están resignificando en su mismo diseño, pero que son consideradas preexistentes e independientes de aquellas.

Sostenemos que en un contexto en el que las desigualdades genéricas se visibilizan y complejizan cada vez con más intensidad en las agendas políticas y sociales se requiere una gestión de la política que visibilice y materialice las prácticas culturales e ideológicas que sostienen y reproducen los estereotipos de géneros. ${ }^{13}$ Aquí es donde las políticas culturales se resignifican centrales para una sociedad que expresa mediante decisiones políticas (Leyes de identidad de Género, Matrimonio Igualitario, Prevención y sanción de la trata de personas y asistencia a sus víctimas, Violencia contra las mujeres, etc. $)^{14}$, la necesidad de transformación de un sistema cultural patriarcal. ${ }^{15}$ Por lo tanto, imbricamos un enfoque crítico para indagar en las relaciones entre la(s) política(s) y el Estado en las formas de diseñar, implementar y evaluar la transversalización de los enfoques de géneros/feminismos por medio de la reflexión de dos niveles de análisis en las políticas públicas del campo cultural. En primer lugar, entonces, explicaremos la vinculación del campo cultural con el contexto político/económico/social a nivel regional y local que enmarcan la noción de diversidad cultural. En un segundo lugar, describiremos las posibilidades y limitaciones de la transversalización de los enfoques de géneros/feminismos que observamos en la política cultural pública reciente por medio de sus planes, programas y proyectos que dieron marco a la agencia de los sujetos inmersos en las diferentes líneas de acción. Por último, daremos cuenta de la falta de coherencia en la gestión interna de dichos programas, planes, proyectos, etc. Asimismo, visibilizaremos la necesidad de planificación de dichas líneas de acción en conjunto con otras políticas sociales que se diseñan e implementan con perspectiva de géneros/feminismos. En este trabajo ejemplificaremos la propuesta mediante la observación en la gestión de algunas líneas de acción que se han llevado a cabo en y desde el Ministerio de Cultura de la Nación Argentina entre los años 2014 y 2015.

\section{La paradoja del binomio cultura-desarrollo como razón de la invisibilización de las problemáticas de géneros/feminismo en las políticas culturales públicas}

La noción de desarrollo, a modo resumido, surge con la finalidad de reducir los estragos económicos y sociales negativos de la Gran Guerra a finales de la Segunda Guerra Mundial. El presidente norteamericano Harry Truman propuso a la 
recién creada Organización de Naciones Unidas (ONU) la noción de desarrollo internacional. Desde la Casa Blanca y como propuesta para las primeras políticas internacionales se concibieron políticas públicas aplicables a nivel internacional que buscaban aliviar la pobreza a nivel mundial y mejorar la calidad de vida de las poblaciones. Esta primera conceptualización apuntaba al desarrollo para obtener mejoras económicas para la población. Cabe señalar que estos eran tiempos de guerra fría, por lo tanto, estas políticas no fueron inocuas pues pretendían tener injerencia directa de los países que hacían contrapeso al naciente bloque soviético, sobre todo en los países del denominado tercer mundo. Desde entonces y hasta ahora las nociones de desarrollo se han modificado de acuerdo a los tiempos, las necesidades y preocupaciones humanas, así como los propios desarrollos tecnológicos, el recambio e intensificación de los flujos de población, el reacomodo de los modos de producción y las pulsiones políticas de los pueblos, por mencionar algunos de los cambios recientes más evidentes (País Andrade y Molina Roldán, 2013).

En otras palabras, desde ese contexto el desarrollo será entendido como una propuesta política que referirá al permanente conjunto de acciones alternativas a una situación inequitativa dada, en búsqueda del crecimiento de las distintas comunidades. Los objetivos de estas acciones siempre serán la igualdad, la inclusión y el bienestar de las sociedades que no lo han podido conseguir debido a las malas acciones de los gobiernos y actores locales de turno (Manzanal, 2006). Este esqueleto cíclico y cambiante - pero no superador- que tiñe las distintas acciones políticas que buscan el desarrollo, generan que para la década de los setenta la noción de desarrollo económico comience a ser cuestionada ya que el desarrollo industrial que estimuló provocó un alto desequilibrio en los sistemas ecológicos del planeta. Se le sumó entonces la noción de sustentable. Para la siguiente década este desarrollo sustentable que seguía teniendo como objetivo obtener bienes materiales cambia su centro de atención y sitúa al ser humano y a las comunidades en el centro del desarrollo. Este giro se aceleró con la publicación del Reporte de Desarrollo Humano del Programa para el Desarrollo de las Naciones Unidas (PNUD) en 1991 cuando “(...) se concibió al desarrollo como la expansión de la capacidad humana como el [...] crecimiento de las capacidades" de las personas para llevar el tipo de vidas que desean, lo cual incluye el acceso a la participación y los recursos culturales (Throsby, 2008: 2). Así, en las convenciones posteriores de 1998 y 2000 la UNESCO reforzó y amplió la vinculación entre desarrollo y cultura. En ambos Informes Mundiales de Cultura se enfatizó la necesidad de crear políticas públicas que tuvieran como ejes ambos elementos. Como consecuencia se han incorporado una nueva serie de conceptos: diversidad, multiculturalidad, sustentabilidad, economía y ciudadanía cultural (País Andrade, 2013).

Lo relevante de este pequeño resumen es que se han generado nuevas tareas y responsabilidades para el sector cultural, a la vez que diferentes actores se resignifican en la relación entre cultura y desarrollo. En la búsqueda de llevar la cultura al centro de las políticas vinculadas al desarrollo, se le concibe como un ente capaz de mejorar la vida de las comunidades mediante la transmisión de formas de vida tradicionales, representaciones distintivas de cultura, ética global, prácticas 
sociales, conocimientos locales, diseminación del conocimiento, creación y preservación del patrimonio humano. Desde entonces, siguiendo estos lineamientos, las políticas regionales y nacionales se han construido desde la visión del denominado desarrollo cultural. Al mismo tiempo, a partir de los años 90, las políticas culturales se transforman conforme a tres ejes: la descentralización, la integración de las artes y las culturas tradicionales y mediáticas a nivel escolar, la inclusión de sectores marginados a través de la ciudadanía cultural (Miller y Yúdice, 2004). ${ }^{16}$

En el marco de estas reformulaciones, el desarrollo ya no se reduce al crecimiento económico, sino que se plantea la necesidad de lograr el desarrollo social, mejorar la equidad, fortalecer la democracia y preservar los equilibrios medioambientales (Kliksberg, 2000). En este sentido, las expresiones identitarias de los grupos sociales -sus producciones y prácticas- son concebidas como elementos diferenciadores en un mercado de bienes simbólicos, los cuales no sólo procuran la cohesión social, sino que se (re)producen como recursos económicos y de acceso a derechos ciudadanos que en muchos casos habían sido negados a colectivos de mujeres, diversidades sexuales, LGBTTTI, entre otros colectivos que se resignifican en nuestro estudio.

De esta forma, las prácticas y estrategias culturales se fueron convirtiendo en espacios relevantes de observación y reflexión en la (re)configuración identitaria de estos grupos y/o sujetos debido a la necesidad de transformaciones culturales/ ideológicas a nivel de lo social y lo político. En la década en estudio esto se resignificó además como necesario ya que las promulgaciones de leyes como Matrimonio igualitario e Identidad de Género (entre otras) suponen macro y micro transformaciones en las prácticas, ideas y saberes cotidianos que se configuran en el campo cultural.

En suma, gestionar la cultura para el desarrollo inclusivo de la ciudadanía se ha vuelto un imperativo en el marco de las políticas nacionales (y también regionales) ${ }^{17}$ de las últimas décadas lo cual reconfigura el estudio del campo cultural y sus políticas en y desde temáticas novedosas. En este contexto se resignifican como objeto de conocimiento los procesos, resultados, impactos y paradojas de las políticas de diversidad cultural, multiculturalismos, interculturalismos como paso fundamental para la implementación de estrategias inclusivas. Asimismo, se convierte en fundamental para mejorar la validez de los planes y recursos que se asignan a dichas acciones como así también la interpelación analítica acerca de la transversalización de los enfoques de mujeres/géneros/feminismos que se hace en ellas. ${ }^{18}$

Ante esto, (re)construir la idea de "desarrollo" a nivel mundial y local de las últimas décadas se tornó imprescindible en estas líneas para entender la formulación y diseño de las políticas culturales en la complejidad del vínculo dado por las demandas de la diversidad cultural y las demandas de los distintos colectivos de mujeres/géneros/feminismos. Así, la posibilidad de sumar a nuestros estudios de políticas públicas a los actores sociales, las prácticas, las producciones y las negociaciones/resistencias entre quienes intervienen en el campo cultural nos permitirá entender el desarrollo en dos procesos dialécticos: como proceso que sostiene las 
desigualdades sociales, económicas, culturales y de género preexistentes -si es que no las ha profundizado-en un sistema capitalista y patriarcal que no pareciera estar siendo cuestionado; y/o paradójicamente, como arena posible de cambio de dichas inequidades. ${ }^{19}$

\section{La paradoja de la transversalización de la cuestión de géneros/ feminismos en las políticas culturales públicas para el desarrollo y la inclusión de las mujeres}

En el marco de los últimos 12 años del proceso político de origen peronista denominado kirchnerismo, desarrollado en Argentina, se diseñaron ciertos programas, proyectos y líneas de acción caracterizados en términos generales, por discursos que rechazaban las miradas del neoliberalismo, las políticas económicas desarrollistas y los tratados del libre comercio. Desde estos enfoques, se promulgaron (con matices, por supuesto) políticas públicas destinadas a la defensa del Mercosur, el alineamiento internacional latinoamericano y la revalorización de los derechos humanos. De esta forma se visibilizaron ciertas tensiones regionales que estaban presentes en relación con el acceso de las minorías étnicas, sexuales, religiosas, económicas; $\mathrm{y}$, de integración de la diversidad cultural (que ya formaban parte de las agendas públicas a nivel local y a nivel regional) materializando políticas y programas locales destinados a operativizar la incorporación de diferentes acciones que respondieran a los lineamientos de positivas garantías internacionales. A los fines de nuestro estudio subrayamos: el Pacto Internacional sobre los Derechos Civiles y Políticos (1966) junto al Pacto Internacional sobre Derechos Económicos, Sociales y Culturales $(1966)^{20}$; y, una serie de resoluciones, declaraciones y exhortaciones en favor de la igualdad de las mujeres que venían siendo sancionadas como instrumentos internacionales de derechos humanos. ${ }^{21}$ En este contexto, surgen y/o se profundizan acciones y programas gubernamentales que resignificarán a ciertas minorías vulneradas como temas de agenda política y; simultáneamente, en poblaciones construidas como discretas, problemáticas, y destinadas a la intervención del Estado (Foucault, 1978): migrantes, indígenas, diversidad sexual, mujeres pobres, etc.

Desde este prisma, las reflexiones acerca de las políticas públicas relacionadas con aquello que puede caracterizarse como derechos culturales y de géneros/feminismos adquirieron en nuestra observación un rol relevante..$^{22}$ En este marco es donde la pregunta ¿cómo se ha gestionado la transversalización de los enfoques de mujeres/géneros/feminismos desde las políticas culturales públicas locales, para la (re)configuración de los derechos de la diversidad cultural /agencias en cuestiones de géneros? construyó sentido.

Como primer paso, para poder responder este interrogante identificamos aquellas prácticas, eventos, líneas de acción y/o programas que dieran cuenta de la incorporación de un enfoque de géneros/feminismos en sus haceres. Para esto relevamos la información que brindaba a simple vista en la página web el Ministerio de Cultura de la Nación Argentina entre los años 2014 y 2015. Hicimos este recorte temporal por ser un hito en la historia de las instituciones de Cultura en 
nuestro territorio ya que por primera vez Cultura se eleva a rango ministerial el 7 de mayo de 2014. ${ }^{23}$

Además, examinamos (solamente) la Secretaría de Políticas Socioculturales (País Andrade, 2014; 2015) y la Subsecretaría de Promoción de Derechos Culturales y Participación Popular (País Andrade, 2014; 2015; 2016) por ser las que -a simple vista- materializaban la perspectiva de géneros/feminismos en algunas de sus acciones de trabajo.

En el marco de lo que se llamó Secretaría de Políticas Socioculturales (creada en 2011) las acciones específicas que incorporaron la perspectiva de género fueron: Cultura Infancia ${ }^{24}$; Café Cultura ${ }^{25}$; Puntos de Cultura ${ }^{26}$; y Derechos Humanos. ${ }^{27}$ Específicamente en su Subsecretaría de Promoción de Derechos Culturales y Participación Popular se llevaron a cabo desde el año 2014 diferentes actividades relacionadas con temáticas asociadas a las desigualdades e inequidades socioculturales. A los fines de este escrito nos interesa subrayar las siguientes actividades: el Panel Tania Hermida: Las mujeres no hablamos solo de mujeres o de género y el panel Mujeres en lucha, ambas realizadas en el marco del Foro Nacional y Latinoamericano por una Nueva Independencia.

Asimismo, desde la Secretaría de Cultura Pública y Creatividad (creada en 2015) pudimos registrar ciertas actividades relacionadas a la temática de géneros -que se suman a los espacios ministeriales relevados- como la charla El patrimonio vivo, desde la perspectiva de género en el marco del Primer Encuentro Nacional de Patrimonio Vivo.

Específicamente en actividades de intervención territoriales, desde el Programa Derechos Humanos dependiente de la Secretaría de Políticas Socioculturales se han llevado a cabo charlas/talleres de Promoción de la Igualdad de Género: Todxs iguales, todxs diferentes en distintas localidades de las provincias argentinas de Entre Ríos y Chaco en el marco de La Semana por la Equidad de Géneros.

A partir de las áreas, programas y acciones identificadas nos interesó reconstruir algunas de las narrativas que resignificaron estos quehaceres para dar cuenta de la transversalización de la perspectiva de mujeres/géneros/feminismos en las temáticas abordadas. A continuación, sintetizaremos, por tanto, diferentes discursos y prácticas identificadas de ciertos actores que fueron parte de las áreas, programas y acciones identificadas: instituciones, investigadores/as, funcionarios/as y talleristas en los años que duraron estas actividades.

Una de las panelistas del encuentro realizado en la capital de la provincia argentina de San Miguel de Tucumán afirmaba: "La cultura de hoy es una cultura de la visualidad que, como nunca antes, irrumpe en lo cotidiano, pero ha devenido en una industria de la subjetivación (...)", es decir, la cultura es "utilizada" para el entretenimiento y el espectáculo como objetivo primero. Esta afirmación parecería estar innovando en una actitud disruptiva de las propias prácticas materializando que las diversas formas del lenguaje (imágenes, palabras, etc.) son formas de (re) conducir poder. En consecuencia, se convertiría en necesidad: “( ...) pensar en otro tipo de mujer que ya no sea la princesa de los melodramas, sino una mujer popular, una mujer como las que venimos escuchando en estos foros (...) Si encontramos 


\section{Cuadro 1 \\ Organigrama del Ministerio de Cultura}

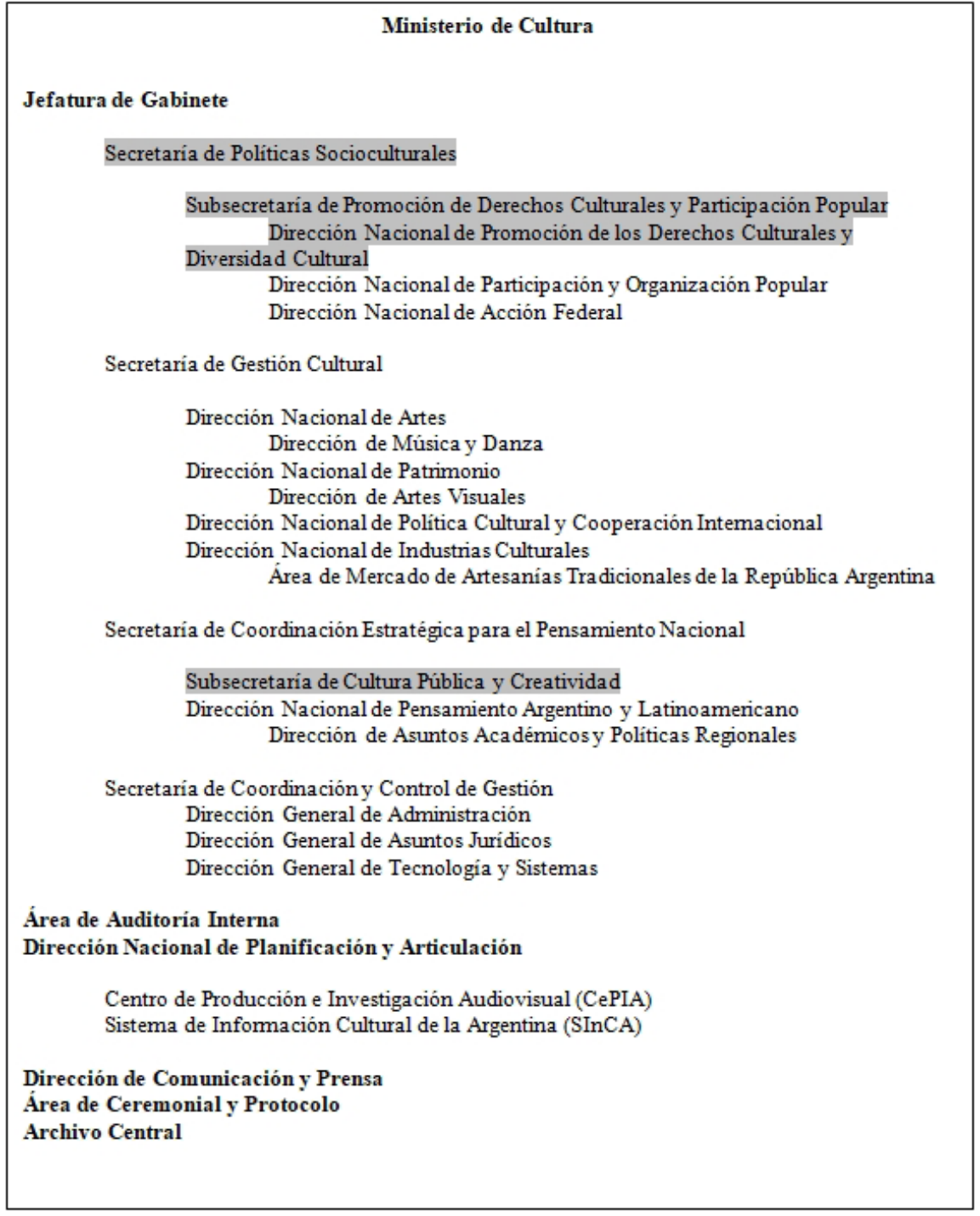

Fuente: elaborado por la autora a partir de los datos extraídos de la página oficial del Ministerio el día 27-04-2015 (se remarcan las áreas en las que se ha focalizado el trabajo de campo). 
ese nuevo género, que nada tiene que ver con la princesa, vamos a encontrar quizá una nueva manera de narrarnos como pueblo latinoamericano" (Panelistas).

Por su lado en la Charla "El patrimonio vivo, desde la perspectiva de género" que se realizó en la Ciudad de Buenos Aires, Rostagnol, afirmaba que: "Cualquier hecho cultural está atravesado por el género" y que la mujer juega un rol primordial en la transmisión generacional del patrimonio. Sin embargo, "Hay actividades que realizan las mujeres que son valoradas por la comunidad, pero que son tradicionales. Las mujeres son vistas como guardianas de una tradición. Ahí hay una doble lectura, porque la tradición también está subvalorada" (Rostagnol, 12-082015). Es decir, la necesidad de visibilizar:

“(...) la tensión de pensar en los derechos culturales” (...) "Muchas veces, se habla de diversidad cultural y se apunta a la igualdad, pero también hablar de diversidad cultural puede estar escondiendo la desigualdad. Y las mujeres están en el medio de esa tensión (...) El desafio, entonces, es mantener relaciones equitativas y lograr que cada comunidad lleve adelante las prácticas que crea que son más adecuadas" (Rostagnol, 12-08-2015).

No obstante, la expositora expresó algunas situaciones en las cuales se produce la paradoja:

"En ocasiones, algunas relaciones inequitativas de género son vistas desde afuera como parte de una cultura, lo que ocurre con las prácticas de mutilación genital femenina en algunas comunidades. En nombre de la diversidad cultural, se dice que hay que preservar esas costumbres y que no se puede intervenir. Tal vez está bien, tal vez está mal, pero creo que se precisa la problematización de estos casos" (Rostagnol, 12-08-2015).

Estas citas nos permiten (someramente) ejemplificar cómo se fue incorporando un discurso con perspectiva de mujeres/géneros/feminismos en las acciones y eventos culturales; además, reflexionar sobre política cultural pública desde esta óptica nos demandó estar atentos/as a las narrativas con las cuales se (re)construyen subjetividades diversas en el marco de las acciones concretas. No obstante, parecería que en dichas narraciones se homogenizan y hegemonizan distintos procesos de desigualdad genérica en el nombre del "respeto y tolerancia" a "lo diverso", naturalizando y/o legitimando desigualdades socioculturales. ${ }^{28}$ Ante esto, y para complejizar los discursos que identificamos en las actividades, entrevistamos a dos trabajadoras de la cultura que estuvieron a cargo de las charlas/talleres enmarcadas en la Semana por la Equidad de Géneros.

En las entrevistas nos contaban que la idea de trabajar las temáticas de género llegó por un contacto personal (una de ellas es militante en Derechos Humanos) en el año 2013 con la directora de una Organización que trabaja Trata de personas con fines de explotación sexual a nivel federal. En esta oportunidad, sin embargo, no se logran unificar objetivos de trabajo ya que la Organización quería llevar a cabo un Gran Evento Cultural ${ }^{29}$ para la visibilización de la problemática y las 
funcionarias del Ministerio querían llevar a cabo acciones que intervinieran directamente en diversos territorios del país. No obstante, queda instalada la inquietud en las trabajadoras del Programa quienes a partir de 2014 plantean en el interior de la Secretaría objetivos y acciones territoriales destinadas a trabajar las diversas temáticas en relación con "la desigualdad de género" (Funcionaria, 19-04-2016, Buenos Aires).

Así, a partir de 2014 con el mismo presupuesto y las mismas diez personas que trabajaban en el Programa se proponen el Taller de Promoción de la Igualdad de Género: "Todxs iguales, todxs diferentes". Con estos talleres y tres personas (de las diez) a cargo, viajaron a diferentes lugares del país donde fueron interactuando con diferentes actores locales y por tanto visibilizando variadas problemáticas: violencia verbal en el noviazgo (y a veces física), violencia a varones gays, mujeres lesbianas, violencia familiar, violencia contra las mujeres por parte de parejas y/o ex parejas, trata de mujeres con fines de explotación sexual, denuncias a las comisarías locales por su actuación en ciertos casos, dificultades de las Comisarías de la Mujer para el trabajo cotidiano en estos temas, etc.

En este marco, se llevaron a cabo las actividades denominadas Semana por la Equidad de Géneros que se realizaron en las provincias de Entre Ríos (del 25 al 28 de agosto de 2015) y de Chaco (del 27 al 31 de julio de 2015), desde donde se trabajaron temáticas contra las violencias, el respeto a la diversidad y la igualdad de derechos. Eran guiadas por la intención de “(...) generar espacios de debate y facilitar la visibilización de las problemáticas sociales derivadas de las redes de explotación humana, y hacer aportes que permitan erradicar los abusos causados por las violencias sociales de cualquier tipo" (Funcionaria, 19-04-2016, Buenos Aires). Las acciones fueron conducidas por medio de charlas, talleres ("Economía del cuidado", "Mujeres libres de violencia", "Otras masculinidades", "El bordado como trazo", "El taller de Promoción de Igualdad de Género y de Comunicación Popular"), proyección de películas y documentales ("Puja, venir al mundo", "No hay Trato") y muestras artísticas ("Ellas quieren contarnos") dirigidas a distintos públicos, en especial a los/as jóvenes. Por tanto, las actividades enmarcadas en este ciclo abordaron temáticas como la mirada y la imagen que tienen y desean de sí mismos/ as, la construcción de la identidad, las diferencias entre los géneros, la diversidad sexual, la trata de personas con fines de explotación sexual y la multiplicidad de violencias que se generan a partir de los estereotipos genéricos. En resumen, “(...) conceptos y temas como sexo, género, patriarcado, heteronormatividad y división social del trabajo fueron objeto de debate y reflexión durante el encuentro" (coordinadora del Programa de Derechos Humanos de la cartera de Cultura nacional, 31/07/2015, Chaco). Todos en estrecha relación con la noción de la equidad de género como derecho humano: "Fue emocionante y conmovedor ver a tantos jóvenes comprometiéndose con esta lucha por los derechos humanos" (Ídem).

Sin embargo, las trabajadoras del programa contaban en situaciones de entrevistas los diferentes obstáculos que tuvieron que enfrentar en las distintas localidades en las que intervinieron: tensiones políticas partidarias locales que se materializaban en muchos casos obstaculizando la tarea (no les daban sonido, no convocaban 
gente, ellas mismas tenían que hacer los contactos institucionales locales para realizar la tarea en escuelas, Centros Culturales, etc.); falta de recursos económicos y humanos, relaciones machistas expresadas en que los referentes locales hombres no se dirigían a las referentes nacionales mujeres para organizar: “(...) aunque el chico que viajaba con nosotras no tenía jerarquía ministerial preferían hablar con él que conmigo que era la representante de Nación" (Coordinadora del Programa, 19-04-2016, Buenos Aires).

Por ende, la gran dificultad que tuvieron para articular con los/as distintos/as actores locales por cuestiones de política partidaria interna y machismos enquistados es una de las grandes deudas que las entrevistadas consideran que quedaron en los territorios de las acciones que llevó a cabo el Programa. Otro desacierto que plantean las trabajadoras es haber pensado que tenían más tiempo y/o que el programa perduraría (a pesar del cambio de gestión política) para continuar con la tarea en la que se habían embarcado entendiendo lo cultural como "(...) espacios de transformación". Suman, además, la “(...) angustiosa sensación de haber realizado acciones directas, haber abierto temas y ahora no tener la posibilidad de trabajarlas, devolverlas en el territorio (...)" (Funcionaria del Programa, 19-042016, Buenos Aires). ${ }^{30}$

Ahora bien, las breves descripciones de diferentes líneas de acción ministeriales transversalizadas con perspectivas de mujeres/géneros/feminismos que acabamos de reseñar nos permite observar cómo la noción de "diversidad" (cultural/sexual) ha sido resignificada y gestionada como una cualidad homogénea y sin conflictos (sobre todo en términos de géneros y sexualidades) -la cual poseen algunas personas y/o grupos- haciendo que las políticas culturales públicas de las últimas décadas hayan sido provistas por la retórica de la multiculturalidad (Fraser, 2009, entre otras/os). Sin embargo, la diversidad cultural no es tan homogénea ni tan disciplinada. Al respecto García Canclini (2005) plantea contradicciones y presupuestos en la diversidad cultural afirmando que existe una paradoja entre cultura y desarrollo. Si por un lado la cultura es pensada como recurso, por otro, es el lugar donde pueden enfocarse los vínculos entre la desigualdad y la penuria. Es decir, por un lado, la cultura como potente motor de desarrollo; por otro, las culturas como pretexto para marcar las diferencias y a menudo para discriminar. Según este autor, tener cultura y desarrollo son actividades complementarias: cultura sin excluir, sino convivir. Desarrollo sin competencia y con solidaridad (Ídem).

Asimismo, dimos cuenta en el apartado anterior de cómo la(s) política(s) del Estado referidas a lo cultural se fueron (re)configurando en un campo en el que operan agentes públicos, privados y comunitarios: políticos y/o funcionarios, burocracias intelectuales, creadores, artistas, empresarios, públicos y asistentes (Ejea, 2011) y también diversos colectivos, movimientos y/o comunidades específicas como las de Derechos Humanos, feminismos, grupos de lesbianas, gays, bisexuales y las personas transgénero e intersexuales, indígenas, migrantes, entre otros. Estas interpelaciones han ido generando a la(s) política(s) cultural(es) públicas como un grupo de acciones complejas y como un espacio institucionalizado por empresas, organismos estatales, fundaciones, $\mathrm{ONG}$, tanto municipales, provincia- 
les como nacionales y mundiales. La inherente presencia de la cultura en el campo político (Giménez, 2007) sustenta que lo cultural no puede considerarse como un momento o un campo de estudio exterior a lo político.

Consecuentemente, por medio de los ejemplos que hemos dado, visibilizamos lo cotidiano de las acciones, planes y proyectos gubernamentales, lo cual nos condujo a identificar y diferenciar los avances/limitaciones que encuentra la transversalización de los enfoques de mujeres/géneros/feminismos en vínculo con el binomio cultura-desarrollo en y desde las políticas culturales públicas locales; y, en y desde los distintos actores intervinientes enmarcados en la noción de diversidad cultural. De esta forma, entendimos la necesidad de observar cómo el sujeto interioriza la cultura y la expresa de diferentes formas, bajo diversas identidades y estrategias identitarias de participación social, donde resignificará y reconfigurará sus derechos en el campo de la diversidad cultural /agencias.

\section{Consideraciones finales}

En este texto, resumimos cómo en las últimas décadas se ha ido configurando la idea de desarrollo a nivel local/regional/mundial para imbricarlo con la formulación de las políticas culturales públicas, diseñadas en la complejidad del vínculo dado por las demandas de la diversidad cultural, en específico con las demandas de los distintos colectivos de mujeres/géneros/feminismos. En esa problematización que construimos observamos la necesidad de sumar a nuestros estudios de políticas culturales públicas a los actores sociales, las prácticas, las producciones y las negociaciones/resistencias entre quienes intervienen en el campo cultural.

Además, los ejemplos que trabajamos (enmarcados en los últimos años de la gestión kirchnerista) nos permitieron visibilizar las problemáticas que se han ido incorporando y/o profundizando -discursiva y territorialmente- desde las políticas culturales públicas en cuestiones de géneros/feminismos por medio de algunas acciones y/o programas en la última década. Esto nos condujo a identificar y diferenciar los avances/limitaciones que encontró la transversalización de los enfoques de mujeres/géneros/feminismos en y desde las políticas culturales públicas locales; $\mathrm{y}$, en y desde los distintos actores intervinientes: por un lado se difundieron y reflexionaron las problemáticas y las cuestiones de géneros/feminismos enfocadas en las mujeres en el campo cultural-artístico-patrimonial; por el otro, se intervino en diferentes territorios con problemáticas de agenda en relación con ser mujer y varón. Paralelamente; mientras que los discursos académicos supusieron interrogantes políticos /conceptuales novedosos al campo cultural, las prácticas territoriales mostraron debilidades políticas/conceptuales en el campo social abriendo y/o profundizando desigualdades existentes. Además de visibilizar, en muchos lugares, temáticas en la agenda local que tensionaron la tarea específica y pusieron en exposición a diversos/as actores locales hasta el momento no cuestionados. Además, identificamos en la gestión de las distintas acciones algunos de los núcleos temáticos en cuestiones de géneros de la agenda feminista pública nacional y las problemáticas territoriales de las personas de carne y hueso: violencias en el noviazgo, violencia a varones gays, mujeres lesbianas, violencia familiar, violencias 
contra las mujeres por parte de parejas y/o ex parejas, trata de mujeres con fines de explotación sexual, denuncias a las comisarías locales por su actuación en ciertos casos, dificultades de las comisarías de la Mujer para el trabajo cotidiano en estos temas, entre otras).

Resumiendo, al observar ciertas áreas específicas ministeriales de cultura pudimos percibir cómo las distintas acciones culturales públicas con perspectiva de mujeres/géneros/feminismos que se han llevado a cabo en la última década en Argentina han interpelado (con matices) a los/as diversos/as actores locales que participan del campo cultural. Si bien han generado la posibilidad de (re)pensarse en y desde las complejidades específicas de la diferencia de géneros, no han logrado transverzalizar los diagnósticos, diseños y ejecuciones de un plan estratégico de cultura pública ya que han sido aisladas y sin vínculos entre sí. Por tanto, la transversalización de los enfoques de mujeres/géneros/feminismos para garantizar la igualdad en el campo cultural nacional implicó que la gestión de los/as diversos/ as actores que participaban en los espacios culturales se llevara a cabo según sus propios intereses, demandas y expectativas. Afirmamos entonces, que la transversalización de la perspectiva de mujeres/géneros/feminismos en las políticas culturales públicas estuvo desvinculada de la producción de las mismas resultando de ello experiencias politizadas (o despolitizadas) de formas desiguales.

En este sentido, señalamos cómo la decisión política del nuevo gobierno votado en 2015 de interrumpir el Programa de Derechos Humanos (por ejemplo) y de las escasas actividades con perspectiva de mujeres/géneros/feminismos que parecieran estar diseñándose en el Plan de cultura nacional, nos enfrenta con nuevos desafíos para pensar cómo serán significados, resistidos, cuestionados y habitados los géneros y las sexualidades en el campo de la diversidad cultural en los próximos años. Además, nos habilita a discutir si será necesaria (como vimos en las acciones y programas ejemplificados) la presencia de diferentes actores pertenecientes a lo que Graciela Di Marco (2011) denominó "Pueblo feminista". Es decir que, en cada espacio institucional, social y/o territorial la posibilidad/imposibilidad de transverzalizar las cuestiones de mujeres/géneros/feminismos queda vinculada a la presencia de actores sociales y/o personas que estén en tema y quieran problematizarlo.

Consecuentemente, proponemos para el estudio de las políticas culturales públicas y su gestión llevar a cabo etnografías de programas, proyectos y/o acciones culturales que logren visibilizar cómo las políticas públicas en el campo cultural son el resultado de matices, encuentros, desencuentros, tensiones, negociaciones y resistencias entre los/as diferentes actores que participan de las acciones socioculturales en lo cotidiano y/o en lo político. Asimismo, se lograría aportar información que mejore los niveles de coordinación y coherencia entre los diferentes programas, planes y proyectos en el campo cultural y sus diálogos con otras políticas socioculturales destinadas a las mismas poblaciones. Esto mostraría también las diferentes experiencias satisfactorias que no son leídas en el nivel macro de las políticas exitosas que, en muchos casos, profundizan desigualdades en torno a las identidades de género y de las diversidades sexuales. Estos diagnósticos permiti- 
rían exigir a nuestros Estados el derecho de la transversalidad de mujeres/géneros/ feminismos en las políticas culturales públicas para garantizar la búsqueda de inclusión en el acceso, producción y vivencia de lo cultural.

\section{Referencias}

1. Este artículo es corolario de la investigación "Política(s) y Género. Configuración de las prácticas e identidades en el escenario nacional actual desde lo cultural y sus vínculos con las desigualdades sociales y de género. Posdoctorado en Ciencias Sociales, Facultad de Ciencias Sociales, Universidad de Buenos Aires, Argentina. 2015-2017. Directora: Graciela Di Marco.

2. Coincidimos con referir a la "Cuestión del género" ya que el término nos permite entender que:(...) el género no es una propiedad de los sujetos ni es un constructo fijo y terminado, condenado a una perpetua repetición (...) Nos impulsa a detectar y explicar cómo los sujetos se en-generan en y a través de una red compleja de discursos, prácticas e institucionalidades, históricamente situadas, que le otorgan sentido y valor a la definición de sí mismos y de su realidad. Ello implica abrir el interrogante acerca de qué, cómo y por qué invisten y negocian, en y a través de estos dispositivos, posiciones y sentidos singulares" (Bonder, 1998: 6).

3. El régimen militar en esos años había puesto en marcha una sistemática y sangrienta represión sobre las prácticas democráticas políticas y sindicales, como sociales y culturales a través del terrorismo de Estado. Dicha situación dejó un saldo histórico de 30.000 desaparecidos/as (secuestrados/as y asesinados/as) y miles de exilados/as.

4. Referimos a "géneros" para desbordar el binomio femenino-masculino e incorporar las identidades Trans que implican la materialización de diversas formas de ser y sentirse "mujeres" o "varones".

5. Con el plural operativizamos la existencia de distintas corrientes teóricas-metodológicas que se encuentran en debate/tensión/acuerdos dentro de la llamada "teoría feminista".

6. El proceso conocido como la "crisis de 2001" devela la capacidad de lucha y organización entre las mujeres, lo cual se materializa al observar: comedores comunitarios, cooperativas de trabajo, redes de cuidado infantil, movimientos "piqueteros", etc.

7. Se lo denominó kirchnerismo debido a quienes han liderado el movimiento: el 25 de mayo de 2003 llegaba al gobierno el Dr. Néstor Kirchner siendo continuado su mandato en 2007 por la Dra. Cristina Fernández de Kirchner hasta el 10 de diciembre del año 2015.

8. La abreviatura LGBTTTI significa lésbico, gay, bisexual, transexual, transgénero, travesti e intersexual; las primeras 3 (LGB) son orientaciones/preferencias sexuales, las siguientes (TT) corresponden a identidades de género; la siguiente $\mathrm{T}$ corresponde a una expresión de género y la intersexualidad corresponde a una condición biológica.

9. Se podría observar el Programa « Manzaneras » como ejemplo de lo dicho.

10. Referimos a enfoques de mujeres/géneros/feminismo para incorporar a los fines de este trabajo las distintas vertientes que han venido reclamando derechos de inclusión ciudadana y que reconfiguran las políticas públicas actuales. Aclaramos, en este sentido, que en este artículo vamos a centrarnos específicamente en reflexionar sobre la inclusión del colectivo "mujeres". No es nuestra intención homogeneizarlas conceptual y/o empíricamente, pero significarlas de esta forma nos permite entender mejor los fines de este artículo. Para profundizar en estas diferenciaciones recomendamos leer (País Andrade, 2018).

11. Entendemos la política cultural pública como una política pública referida a lo que se entiende por "cultura" en un momento y espacio determinado. La misma se delimita en y desde el conjunto de acciones de quienes detentan el poder estatal dirigido a reforzar, rechazar, confrontar y/o negociar prácticas referidas a lo cultural entre los diversos grupos sociales. Paralelamente, son acciones de y desde los diversos grupos sociales dirigidas a reforzar, rechazar, confrontar y/o negociar prácticas identitarias. Por tanto, la política (socio) cultural es un proceso político-identitario que se va reconstruyendo en una permanente complejidad dialéctica entre quienes detentan el poder del Estado y los diversos actores del campo cultural en un momento y lugar específico. Dar cuenta de este proceso permite visibilizar la 
noción de cultura reconfigurada por cada grupo, lugar, momento y actor social como así también los sentidos y significados que se le otorga a lo cultural (País Andrade, 2016).

12. Referimos al término agencias partiendo de la idea de agente, desde Max Weber, quien entiende que: “(...) los actores sociales actúan intersubjetivamente respondiendo según las formas de actuar de los 'otros', en concordancia con sus propios intereses y de acuerdo con sus cosmovisiones y sus valores culturales" (Catullo 2006: 42). En este sentido, la cultura es “(...) una sección limitada de la infinitud desprovista del sentido del acaecer universal, a la cual los seres humanos otorgan sentido y significado" (ídem).

13. Pensamos en las características de "lo femenino" y "lo masculino" que naturalizan desigualdades sociales amparadas en las diferencias anatómicas de las personas y que se (re)producen/tensionan en las diferentes performances corporales de los grupos trans, campañas contra la violencia sexual, homofóbica, etc., muestras teatrales, fotográficas, televisivas, interacciones y denuncias a través de las redes sociales, etc.

14. Para profundizar en estas leyes se sugieren los siguientes links: Ley 26.743. Ley de Identidad de Género, Disponible en: http://servicios.infoleg.gob.ar/infolegInternet/anexos/195000-199999/197860/ norma.htm. Ley 26.618 y Decreto 1054/10. Ley de Matrimonio Igualitario. Código Civil. Matrimonio Civil. Su modificación. Disponible en: https://soydondenopienso.wordpress.com/2010/07/22/ley-26618-y-decreto-105410-ley-de-matrimonio-igualitario/ Ley 26.842. Ley de Prevención y sanción de la trata de personas y asistencia a sus víctimas, Disponible en:

http://www.acnur.org/t3/fileadmin/Documentos/BDL/2014/9763.pdf. Ley 26.485. Protección Integral para Prevenir, Sancionar y Erradicar la Violencia contra las Mujeres en los Ámbitos en que Desarrollen sus Relaciones Interpersonales. Disponible en: http://feim.org.ar/2017/05/09/ley-26-485-proteccionintegral-para-prevenir-sancionar-y-erradicar-la-violencia-contra-las-mujeres-en-los-ambitos-en-quedesarrollen-sus-relaciones-interpersonales/

15. Desde las ciencias sociales existe cierto acuerdo en utilizar el término patriarcado para dar cuenta de la organización social humana desde la instalación de las religiones judía-cristiana e islámica y su profundización a través del feudalismo. Esta forma de consolidación social se caracteriza por la hegemonía del señor feudal y la iglesia católica, y encuentra analogía en la autoridad del patriarca donde los varones eran reconocidos por la comuna como cabeza de familias. En otras palabras, desde tiempos lejanos las construcciones de género de nuestras sociedades occidentales han producido y (re)producido a través de la historia diferentes espacios y roles sociales de presencia/ausencia que conformaron diferencias y desigualdades entre hombres y mujeres.

16. Para profundizar en las características que tomaron en Argentina las políticas culturales de la década recomendamos leer el estudio de caso del Programa Cultural en Barrios (País Andrade, 2011).

17. Pensemos en los procesos políticos, sociales y culturales que se han ido configurando en toda América latina, en muchos casos expresados en las reformulaciones de las propias Constituciones nacionales como ejemplifican Ecuador y Bolivia.

18. En la región se pueden observar estos procesos en los surgimientos de los feminismos indígenas, comunitarios y/o populares, por ejemplo. En este sentido se recomienda la lectura de los siguientes textos: "Feminismos desde Abya Yala. Ideas y Proposiciones de las Mujeres de 607 Pueblos de Nuestra América" de Francesca Gargallo Celantani (2014); "La guerra contra las mujeres" de Rita Segato (2016); "Feminismos Populares. Pedagogías y Políticas" de Claudia Korol (2016), entre otros.

19. En este sentido -y en otros trabajos (Benedetti y otras, 2013)-, se ha analizado cómo las relaciones entre el Estado y el espacio cultural reconocen un vasto tratamiento en las ciencias sociales en relación con la producción, circulación, consumo, gestión y patrimonialización en el contexto del desarrollo y la diversidad.

20. Pacto Internacional de Derechos Civiles y Políticos: Disponible en http://www2.ohchr.org/spanish/law/ccpr.htm; Pacto Internacional de Derechos Económicos, Sociales y Culturales: Disponible en http://www2.ohchr.org/spanish/law/cescr.htm.

21. La Carta Internacional de Derechos Humanos está compuesta por La Declaración Universal de Derechos Humanos, el Pacto Internacional de Derechos Civiles y Políticos y sus dos protocolos facultativos, el Pacto Internacional de Derechos Económicos, Sociales y Culturales. Asimismo, a través de 
la ratificación de los tratados internacionales, los Estados asumen las obligaciones de respetar, proteger y realizar la totalidad de los derechos humanos consignados en los tratados. Ahora bien, "ambos derechos" (DCPP y el DESC) son parte de un todo (DD.HH.) estando presentes en la Declaración Universal de Derechos Humanos y representan para el Estado obligaciones positivas; requieren de la ejecución de diversas acciones por parte del Estado; obligan a los Estados a adoptar medidas inmediatas para darles efectividad, aun cuando el Estado tenga escasez de recursos; son concebidos como derechos exigibles por aquellos que adscriben a visiones del Estado como Estado mínimo en el marco del derecho privado clásico; podemos decir que los DESC mantienen diferencias de grado y no sustanciales con los derechos civiles y políticos. Es decir, en su conjunto todos los derechos humanos suponen la creación de condiciones institucionales y un complejo de obligaciones positivas y negativas por parte del Estado.

22. Consecuentemente, nuestras investigaciones vienen profundizando los estudios sobre las políticas públicas argentinas desde una perspectiva de géneros (del Río Fortuna y otras, 2013) destinadas a construir cultura como recurso económico, político y/o simbólico (Autora; 2015, 2016).

23. El Ministerio de Cultura de la Nación Argentina se crea el 7 de mayo de 2014 y se designa a Teresa Adelina Sellares, conocida como Teresa Parodi (cantautora argentina) como Ministra del mismo. Hasta la fecha de su creación había funcionado como una Secretaría. La función del Ministerio de Cultura de la Nación era planificar y ejecutar estrategias para la promoción, rescate, preservación, estímulo, acrecentamiento y difusión, en el ámbito nacional e internacional.

24. Programa orientado a lograr que los niños y las niñas sean parte de los procesos culturales. La propuesta no trata solamente de garantizar el acceso a una cultura de calidad o hacerlos partícipes de las políticas culturales planificadas; busca, además, fundar una alianza estratégica con los chicos y las chicas para construir en conjunto la cultura que queremos.

25. El programa nació en 2005, con la intención de multiplicar espacios de debate en toda la Argentina. El punto de partida es la certeza de que el diálogo y la reflexión son herramientas fundamentales e imprescindibles para construir una sociedad más plural e inclusiva. El objetivo sencillo, pero ambicioso: a través de encuentros y conferencias gratuitos, intelectuales, funcionarios/as, científicos/as, artistas, economistas y periodistas de todo el país comparten y ponen en discusión sus opiniones, experiencias y conocimientos ante el público, en torno a una amplia diversidad de temáticas culturales, sociales, educativas, políticas y económicas, buscando interacción y reflexión conjunta. Los ejes centrales de debate de 2014 fueron: medios de comunicación; economía y fondos buitres; rol y políticas de Estado; inclusión; identidad; energía; derechos humanos; género y cultura. Además, para acompañar y fortalecer los procesos de transformación social del país, el programa apunta a generar actividades culturales junto con Casas del Bicentenario, centros culturales, Puntos de Cultura, bibliotecas populares, sociedades de fomento y clubes, entre otros organismos, para así llegar a todos los puntos del país, de un modo horizontal y descentralizado. En 2014 se incorporan dos novedades: el ciclo Ocho mujeres. Cinturas cósmicas del sur, que proponía reflexionar a través del teatro y la poesía latinoamericanos, y homenajear a las mujeres escritoras de América latina; y las Revistas Orales, ediciones multidisciplinarias en soporte digital, donde se trataban asuntos de actualidad nacional, como diálogo interreligioso y género.

26. El Programa Puntos tiene como objetivo promover la organización popular a través de la cultura comunitaria. Las organizaciones seleccionadas para formar parte del Programa reciben apoyo económico y equipamiento multimedia para registrar sus acciones y producir materiales de comunicación. Además, forman parte de una red federal de organizaciones socioculturales de intercambio y cooperación de experiencias de desarrollo comunitario. Esta Red Nacional de Puntos de Cultura está integrada por 250 organizaciones sociales y comunidades indígenas de todo el país, que fueron seleccionadas en las dos primeras convocatorias del Programa, realizadas en 2011 y 2012.

27. El Programa tiene como objetivo promover prácticas de participación que, desde una perspectiva integral de los derechos humanos, permitan reflexionar acerca de las problemáticas presentes en nuestra sociedad. A través de charlas, talleres y muestras, se busca concientizar acerca de la trata de personas, la equidad de género, la erradicación de la violencia institucional y el trabajo infantil; fortalecer el accionar de los organismos de Derechos Humanos nacionales, en pos de alcanzar "memoria, verdad y justicia", recuperando las historias de vida de los/as detenidos/as desaparecidos/as, y prestar apoyo a las organizaciones sociales orientadas a la promoción de estos objetivos. Estas iniciativas permiten 
facilitan la comprensión de los derechos culturales, que constituyen aspectos esenciales para la construcción de una sociedad más justa y democrática.

28. Es decir, no se subrayan las diferencias económicas, culturales y/o sexuales existentes entre el propio colectivo "mujeres"; como tampoco se incluyen las complejidades de las personas cuyos cuerpos han sido feminizados (travestis, trans, etc.), ni aparecen los estudios y observancias de los colectivos de "varones" (no se complejiza la necesidad de pensar también otros tipos de masculinidades para ser resignificadas en las posibles transformaciones culturales actuales), por ejemplo.

29. La espectacularización de la cultura es una característica presente en la gestión de gobierno actual.

30. Una de las direcciones que pensaban tomar en 2016, luego del diagnóstico territorial, refería a intervenir en menos territorios y centrarse en los lugares donde más dificultades concretas habían detectado. No obstante, la gestión cambió y con ello el Programa desapareció.

\section{Bibliografía}

Abéles, M. (1997). La antropología política: nuevos objetivos, nuevos objetos. Revista Internacional de Ciencias Sociales, N 53: Antropología - Temas y Perspectivas: I. más allá de las lindes tradicionales. Recuperado de http://www.unesco.org/issj/

Aguilar, P. L. (2011). La feminización de la pobreza: conceptualizaciones actuales y potencialidades analíticas. Revista Katálysis, Vol. 14 (1), Brasil: Universidade Federal de Santa Catarina Santa Catarina, 126-133.

Anzorena, C. (2013). Mujeres en la trama del Estado: Una lectura feminista de las políticas públicas. Mendoza. Argentina: Ediunc.

Benedetti, C., Cardini, L. y País Andrade, M. (2013). Estado, políticas públicas y cultura en tres experiencias de investigación socioantropológica. Actas de las Séptimas Jornadas de Investigación en Antropología Social. Facultad de Filosofía y Letras. Universidad de Buenos Aires. Buenos Aires. Argentina. Recuperado de http://www.aacademica.com/000-063/117.pdf

Bonder, G. (1998). Género y Subjetividad: avatares de una relación no evidente. En Montecino, S. y Obach. A. (Ed.). Género y Epistemología. Mujeres y disciplinas. (PIEG), Santiago de Chile. Chile: Universidad de Chile, 9-46.

Catullo, M. R. (2006). Ciudades relocalizadas. Una mirada desde la antropología Social, Buenos Aires. Argentina: Biblos.

Del Río Fortuna, C., González Martin, M. y País Andrade, M. (2013). Políticas y género en Argentina. Aportes desde la antropología y el feminismo. Revista Encrucijadas. Revista Crítica de Ciencias Sociales, Junio, No 5. Salamanca. España: Universidad de Salamanca, 54-65.

Di Marco, G. (1997). Las mujeres y la política en los '90. En Schmukler, B. y Di Marco, G. (1997). Madres y democratización de las familias en la Argentina contemporánea, Buenos Aires. Argentina: Biblos.

Di Marco, G. (2003). Movimientos sociales emergentes en la sociedad argentina y protagonismo de las mujeres. Segunda época, volumen VIII, 15-36.

Di Marco, G. (2005). Democratización de las familias. Estrategias alternativas para la implementación de programas sociales. Buenos Aires. Argentina: Baudino Editores.

Di Marco, G. (2011). El Pueblo Feminista. Movimientos sociales y lucha de las mujeres en torno a la ciudadanía. Buenos Aires. Argentina: Biblos.

Ejea, T. (2011). Poder y creación artística en México. Un análisis del Fondo Nacional para la Cultura y las Artes (FONCA). México DF. México: Universidad Autónoma Metropolitana Azcapotzalco.

Falquet, J. (2003). Mujeres Feminismo y Desarrollo. Mujeres feminismo: un análisis crítico de las políticas de las instituciones internacionales. En Desacatos, primavera $\mathrm{N}^{\circ} 11$. México: Centro de Investigaciones y Estudios Superiores en Antropología Social, 13-35.

Foucault, M. (1978). La «gouvemementalité». Curso del College de France, año 1977-1978. «Seguridad, territorio y población», $4^{\circ}$ lección, 1 de febrero de 1978, Aut-Aut, $N^{\circ}$ 167-168, 12-29.

Fraser, N. (2009). Feminism, Capitalism and the Cunning of History. New Left Review, 56.

García Canclini, N. (2005). Todos tienen cultura. ¿Quiénes pueden desarrollarla? Conferencia para el 
Seminario sobre Cultura y Desarrollo en el Banco Interamericano de Desarrollo. Washington. EE.UU.: Georgetown University.

Gargallo Celantani, F. (2014). Feminismos desde Abya Yala. Ideas y Proposiciones de las Mujeres de 607 Pueblos de Nuestra América. México DF, México: Ed. Corte y Confección.

Giménez, G. (2007). Estudios sobre la cultura y las identidades sociales. México DF. México: CONACULTA/ITESO.

Grassi, E. (2003). Políticas y problemas sociales en la sociedad neoliberal. La otra década infame. Buenos Aires. Argentina: Editorial Espacio.

Grassi, E. (2009). Políticas sociales y género: una problematización del concepto de exclusión y la participación social de las mujeres. En Leituras de Resistencia. Corpo, Violencia e Poder (vol. II), compilado por C.S. Tornquist et al. Florianopolis. Brasil: Ed. Mulheres, 241-267.

Jelin, E. (2009). Género y familia en la política pública: una perspectiva comparativa Argentina-Suecia. Interseçoes: Revista de Estudos Interdisciplinares (Rio de Janeiro), año 11, $\mathrm{N}^{\circ} 2,35-55$.

Jelin, E. ed. (1990). Women and social change in Latin America. Londres. Reino Unido: Zed Books.

Kliksberg, B. (2000). El rol del capital social y de la cultura en el proceso de desarrollo. En Kliksberg, B. y Tomassini, L. (Comps.). Capital social y cultura: claves estratégicas para el desarrollo. Buenos Aires. Argentina: Fondo de Cultura Económica.

Korol, C. (2016). Feminismos Populares. Pedagogías y Políticas, Buenos Aires. Argentina: Ed.Chirimbote/ Ed América Libre/ Ed. El Colectivo.

Manzanal, M. (2006). Regiones, territorios e institucionalidad del Desarrollo Local. En Manzanal, M., Neiman, G. y Lattuada, M. (comps.). Desarrollo rural. Organizaciones, instituciones y territorios. Buenos Aires, Argentina: Ciccus, 21-50.

Merchán, C. (2001). La participación de la mujer en la dirección del movimiento de resistencia. Posibilidades y obstáculos actuales. Ponencia (edición digital, dic.2001). Recuperado de http://www. rebelion.org/docs/4859.pdf

Miller, T. y Yúdice, G. (2004). Política cultural. Barcelona, España: Gedisa.

País Andrade, M. (2011).Cultura, Juventud, Identidad: una mirada socioantropológica del Programa Cultural en Barrios, Buenos Aires, Argentina: Estudios Sociológicos Editora.

País Andrade, M. y Molina Roldán, A. (Comp.). (2013). Cultura y desarrollo en América latina. Actores, estrategias, formación y prácticas. Buenos Aires. Argentina: Ediciones Cooperativas / México: De la Vega editores.

País Andrade, M. (2014). Lo cultural' desde una perspectiva de género. Políticas, Desarrollo y Diversidad. Revista Horizontes Sociológicos. Revista de la Asociación Argentina de Sociología (ALAS), Publicación Internacional de Ciencias Sociales, Año 2, $\mathrm{N}^{\circ} 4,126-138$.

País Andrade, M. (2015). Avances y limitaciones en la política cultural argentina y su gestión desde una perspectiva de género. En \#PensarLaCulturaPública. Apuntes para una cartografia nacional, Subsecretaría de Cultura Pública y Creatividad, Ministerio de Cultura de la Nación. Buenos Aires. Argentina, 18-35.

País Andrade, M. (2016). Identidades culturales en y desde las fronteras. Un enfoque de género a la(s) políticas y a la(s) práctica(s) culturales juveniles. Buenos Aires. Argentina: Antropofagia.

País Andrade, M. (Compiladora) (2018). Experiencias interdisciplinarias de intervención/investigación desde una perspectiva de géneros. Buenos Aires. Argentina: CICUUS (En prensa).

Rauber, I. (2002). Mujeres Piqueteras: el caso de argentina.Genève: iuéd-efi; Paris: L'Harmattan. Recuperado de http://graduateinstitute.ch/files/live/sites/iheid/files/sites/genre/shared/Genre_ docs/2887_Actes2002/07_rauber.pdf, pp. 159-165.

Rodríguez Gustá, A. L. y Caminotti, M. (2010). Políticas públicas de equidad de género: las estrategias fragmentarias de la Argentina y Chile. Revista SAAP, 4 (1). Recuperado http://www.scielo.org.ar/ scielo.php?script=sci_arttext\&pid=S185319702010000100003\&lng=es\&tlng=es

Segato, R. (2016). La guerra contra las mujeres. Madrid. España: Traficantes de Sueños.

Shore, C. y Wright, S. (1997). Introduction: Policy. A new field of anthropology. Anthropology of Policy: Critical Perspectives on Governance and Power. London. United Kingdom: Routledge, 3-39. Throsby, D. (2008). Culture in Sustainable Development: Insights for the future implementation of 
art. 13 Convention on the Protection and Promotion of the Diversity of Cultural Expressions, Sidney. Recuperado de http://unesdoc.unesco.org/images/0015/001572/157287e.pdf

Wedel, J., Shore, C., Feldman, G. and Lathrop, S. (2005). Toward an Anthropology of Public Policy. In The ANNALS of the American Academy of Political and Social Science, 30-51. Recuperado de http:// ann.sagepub.com/cgi/content/abstract/600/1/30

Wittig, M. (1986). The Mark of Gender. In Feminist Issues 5.2 (1985), 3-12.

\section{Páginas web consultadas}

Secretaría de Políticas Socioculturales:

http://www.cultura.gob.ar/areas/subsecretaria-de- politicas-socioculturales/

Ministerio de Cultura de la Nación: http://www.cultura.gob.ar/

Recibido: 20/02/17. Aceptado: 29/05/2017.

Marcela País Andrade, "La transversalización del enfoque de géneros en las políticas culturales públicas: el caso del Ministerio de Cultura argentino". Revista Temas y Debates. ISSN 1666-0714, año 22, número 35, enero-junio 2018, pp. 161-180. 